

\title{
Tamely ramified Iwasawa modules having no non-trivial pseudo-null submodules
}

\author{
par Tsuyoshi ITOH
}

\begin{abstract}
RÉsumé. Ce travail fait suite à l'article [4] de Satoshi Fujii et l'auteur. Soient $k$ un corps de nombres, $p$ un nombre premier, et $k^{c} / k$ la $\mathbb{Z}_{p}$-extension cyclotomique. Pour un ensemble fini $S$ de nombres premiers qui ne contient pas $p$, le module d'Iwasawa (par rapport à la pro- $p$ extension abélienne maximale non ramifiée en dehors de $S$ ) a été étudié dans plusieurs articles. Nous donnons des exemples non-triviaux où $X_{S}\left(k^{c}\right)$ a un sous-module fini non-nul avec $k$ totalement réel. Nous donnons également un exemple similaire dans le cas de la $\mathbb{Z}_{p}^{\oplus 2}$-extension d'un corps quadratique imaginaire. De plus, nous discutons en appendice des analogues faibles de la conjecture de Greenberg pour $X_{S}\left(k^{c}\right)$.
\end{abstract}

ABstract. The present paper is a sequel to the previous paper [4] (by Satoshi Fujii and the author). Let $k$ be an algebraic number field, $p$ a prime number, and $k^{c} / k$ the cyclotomic $\mathbb{Z}_{p}$-extension. For a finite set $S$ of prime numbers which does not contain $p$, the Iwasawa module $X_{S}\left(k^{c}\right)$ (with respect to the maximal pro- $p$ abelian extension unramified outside $S$ ) has been studied in several papers. We will give some non-trivial examples such that $X_{S}\left(k^{c}\right)$ has no non-trivial finite submodules even when $k$ is totally real. We also give a similar example for the case of the $\mathbb{Z}_{p}^{\oplus 2}$-extension of an imaginary quadratic field. Moreover, weak analogs of Greenberg's conjecture for $X_{S}\left(k^{c}\right)$ are also discussed in the appendix.

\section{Introduction and results}

Let $p$ be a prime number, $S$ a finite set of prime numbers which does not contain $p$. For an algebraic extension $\mathcal{K} / \mathbb{Q}$, let $L_{S}(\mathcal{K}) / \mathcal{K}$ be the maximal abelian (pro-)p-extension unramified outside $S$. We put $X_{S}(\mathcal{K})=$ $\operatorname{Gal}\left(L_{S}(\mathcal{K}) / \mathcal{K}\right)$. When $\mathcal{K}$ is an algebraic number field (i.e., $\mathcal{K} / \mathbb{Q}$ is finite), $L_{S}(\mathcal{K}) / \mathcal{K}$ is finite because all ramified primes are tamely ramified.

Manuscrit reçu le 14 mars 2017, révisé le 12 janvier 2018, accepté le 19 janvier 2018.

2010 Mathematics Subject Classification. 11R23.

Mots-clefs. Iwasawa modules, non-existence of non-trivial pseudo-null submodules.

The author would like to thank Yasushi Mizusawa and Satoshi Fujii. This research was motivated by Mizusawa's comment, and discussions with Fujii gave many suggestions to the author. Moreover, the referee's remarks were useful for improving the contents. The author also would like to thank him/her. This research was partly supported by JSPS KAKENHI Grant Number JP15K04791. 
Let $k$ be an algebraic number field, and $K / k$ a $\mathbb{Z}_{p}$-extension. We put $\Lambda_{K / k}=\mathbb{Z}_{p} \llbracket \operatorname{Gal}(K / k) \rrbracket$. Then, we can show that $X_{S}(K)$ is a finitely generated torsion module over $\Lambda_{K / k}$ (this is often called a tamely ramified Iwasawa module). We will consider the existence of a non-trivial pseudo-null $\Lambda_{K / k}$-submodule of $X_{S}(K)$. (For the definition of pseudo-nullity, see, e.g., [18]. In this case, a pseudo-null $\Lambda_{K / k}$-module is just a finite $\Lambda_{K / k}$-module.) We denote by $k^{c} / k$ the cyclotomic $\mathbb{Z}_{p}$-extension.

In [4], it was shown that if $p$ is odd and $X_{S}\left(\mathbb{Q}^{c}\right) \neq 0$, then $X_{S}\left(\mathbb{Q}^{c}\right)$ always contains a non-trivial finite submodule. On the other hand, when $p=2$, Mizusawa's result [16, Theorem 7.3] implies the existence of the case that $X_{S}\left(\mathbb{Q}^{c}\right) \cong \mathbb{Z}_{2}$ as a $\mathbb{Z}_{2}$-module. Hence, the case when $p=2$ is more complicated. Our first result is a determination of the set $S$ of odd prime numbers such that $X_{S}\left(\mathbb{Q}^{c}\right)$ does not contain a non-trivial finite submodule for $p=2$ (the proof will be given in Section 3).

Theorem 1.1. Assume that $p=2$. Let $S$ be a non-empty finite set of odd prime numbers. For an odd prime number $q$, we denote by $P(q)$ the number of primes in $\mathbb{Q}^{c}$ lying above $q$. (Note that $q$ is finitely decomposed in $\mathbb{Q}^{c}$.) Then $X_{S}\left(\mathbb{Q}^{c}\right)$ does not have a non-trivial finite $\Lambda_{\mathbb{Q}^{c} / \mathbb{Q}^{-s u b m o d u l e}}$ if and only if $S=\left\{q_{1}, \ldots, q_{r}\right\}$ satisfies $q_{1} \equiv \cdots \equiv q_{r} \equiv 3(\bmod 4)$ and $P\left(q_{1}\right)=\cdots=P\left(q_{r}\right)$ (where $q_{1}, \ldots, q_{r}$ are distinct prime numbers).

When $p$ is an odd prime number, we can also find an example of a totally real number field $k$ such that $X_{S}\left(k^{c}\right)$ does not contain a non-trivial finite submodule. Our second result is a simple criterion whether $X_{S}\left(k^{c}\right)$ has no non-trivial finite submodules for a real quadratic field $k$ and certain $p$ and $S$. We denote by $|A|$ the number of elements of a finite set $A$.

Theorem 1.2. Let $p$ be an odd prime number, and $k$ a real quadratic field. Assume that $p$ is inert in $k$ and $p$ does not divide the class number of $k$. Take distinct prime numbers $q_{1}, \ldots, q_{r}$ such that $q_{i} \equiv-1(\bmod p)$ and $q_{i}$ is inert in $k$ for $i=1, \ldots, r$. We put $S=\left\{q_{1}, \ldots, q_{r}\right\}$. We denote by $P\left(q_{i}\right)$ the number of primes of $\mathbb{Q}^{c}$ lying above $q_{i}$, and by $P^{\prime}$ the largest number of $P\left(q_{i}\right)$ for $i=1, \ldots, r$. Then $X_{S}\left(k^{c}\right)$ does not have a non-trivial finite $\Lambda_{k^{c} / k^{-}}$-submodule if and only if

$$
\left|X_{S}(k)\right|=p^{r-1} \cdot\left(\prod_{i=1}^{r} P\left(q_{i}\right)\right) / P^{\prime} .
$$

(Note that $p^{r-1} \cdot\left(\prod_{i=1}^{r} P\left(q_{i}\right)\right) / P^{\prime} \leq\left|X_{S}(k)\right| \leq p^{r} \cdot \prod_{i=1}^{r} P\left(q_{i}\right)$ in this case. We also see that $X_{S}\left(k^{c}\right)$ is infinite if $|S| \geq 2$.)

This theorem will be shown in Section 4. As a consequence, one can find an explicit example such that $X_{S}\left(k^{c}\right)$ does not have a non-trivial finite submodule (see Remark 4.3). We note that when $p$ splits in a real quadratic 
field $k$, the same type result does not hold (see Appendix A). We also give another (non-trivial) example of a totally real field $k$ such that $X_{S}\left(k^{c}\right)$ does not have a non-trivial finite submodule (Proposition 4.5).

Next, we will consider the case of the $\mathbb{Z}_{p}^{\oplus 2}$-extension of an imaginary quadratic field $k$. Concerning this paragraph, see also [4] for the details. Let $\widetilde{k} / k$ be the unique $\mathbb{Z}_{p}^{\oplus 2}$-extension. We put $\Lambda_{\widetilde{k} / k}=\mathbb{Z}_{p} \llbracket \operatorname{Gal}(\widetilde{k} / k) \rrbracket$. Then $X_{S}(\widetilde{k})$ is a finitely generated torsion $\Lambda_{\widetilde{k} / k}$-submodule. In [4], some sufficient conditions such that $X_{S}(\widetilde{k})$ has a non-trivial pseudo-null $\Lambda_{\widetilde{k} / k}$-submodule were given. However, there is a non-trivial example such that $X_{S}(\widetilde{k})$ does not contain a non-trivial pseudo-null submodule. In Section 5, we will prove the following:

Theorem 1.3. We put $k=\mathbb{Q}(\sqrt{-3})$ and $p=3$. Let $\widetilde{k} / k$ be the unique $\mathbb{Z}_{3}^{\oplus 2}$-extension. Take a set $S=\left\{q_{1}, q_{2}\right\}$ of distinct prime numbers which satisfy $q_{i} \equiv 2(\bmod 3)$ and $q_{i}$ is not decomposed in $\mathbb{Q}^{c}$ for $i=1,2$. Then $X_{S}(\widetilde{k})$ is not pseudo-null, and it does not contain a non-trivial pseudo-null submodule.

In Appendix A, we consider analogs of weak forms of Greenberg's conjecture in the sense of Nguyen Quang Do.

\section{Preliminaries}

We shall define some notations. Let $|\cdot|_{p}$ be the multiplicative $p$-adic absolute value normalized as $|p|_{p}=p^{-1}$. In the following of this section, $k$ denotes an arbitrary algebraic number field. We denote by $O_{k}$ the ring of integers of $k$, and by $E(k)$ the group of units of $k$. For a non-zero integral ideal $\mathfrak{m}$ of $k$, we put $R(k, \mathfrak{m})=\left(O_{k} / \mathfrak{m}\right)^{\times} \otimes_{\mathbb{Z}} \mathbb{Z}_{p}$. We consider every algebraic extension field over $\mathbb{Q}$ as a subfield of $\mathbb{C}$, and put $\zeta_{n}=e^{2 \pi i / n}$ for a positive integer $n$.

Let $K / k$ be a $\mathbb{Z}_{p}$-extension. Put $\Gamma=\operatorname{Gal}(K / k)$, and $\Lambda_{K / k}=\mathbb{Z}_{p} \llbracket \Gamma \rrbracket$. We also note that $\Lambda_{K / k}$ is isomorphic to the power series ring $\mathbb{Z}_{p} \llbracket T \rrbracket$ (and we fix an isomorphism). Let $S$ be a finite set of prime numbers not containing $p$. In this case, there is a pseudo-isomorphism

$$
X_{S}(K) \rightarrow \bigoplus_{i=1}^{m} \mathbb{Z}_{p} \llbracket T \rrbracket / p^{c_{i}} \mathbb{Z}_{p} \llbracket T \rrbracket \oplus \bigoplus_{j=1}^{n} \mathbb{Z}_{p} \llbracket T \rrbracket / g_{j}^{d_{j}} \mathbb{Z}_{p} \llbracket T \rrbracket,
$$

where $c_{i}, d_{j}$ are positive integers and $g_{j}$ is an irreducible distinguished polynomial for each $i, j$ (see, e.g., [18], [26]). We put

$$
\mu_{K / k, S}=\sum_{i=1}^{m} c_{i} \quad \text { and } \quad F_{K / k, S}(T)=\prod_{j=1}^{n} g_{j}^{d_{j}} .
$$


$F_{K / k, S}(T)$ is called the characteristic polynomial of $X_{S}(K)$ (in the sense of $\left[18,(5.3 .9)\right.$ Definition]). Note that $\mu_{K / k, S}=0$ if and only if $X_{S}(K)$ is finitely generated as a $\mathbb{Z}_{p}$-module. In particular, if $k / \mathbb{Q}$ is an abelian extension and $K=k^{c}$ (the cyclotomic $\mathbb{Z}_{p}$-extension), then $\mu_{k^{c} / k, S}=0$ (see, e.g., $\left[10\right.$, p. 1494]). Note also that if $X_{S}(K)$ is finite, then $\mu_{K / k, S}=0$ and $F_{K / k, S}(T)=1$. Moreover, we denote by $X_{S}(K)^{\Gamma}$ the $\Gamma$-invariant submodule of $X_{S}(K)$, and by $X_{S}(K)_{\Gamma}$ the $\Gamma$-coinvariant quotient of $X_{S}(K)$ (similar notations will be used in Section 5 under a slightly different setting).

The following is our main criterion. (This type result seems well known, however, we will give a brief proof.)

Proposition 2.1. Assume that there is only one prime $\mathfrak{p}$ of $k$ lying above $p$, and $\mathfrak{p}$ is totally ramified in $K / k$. Then $X_{S}(K)$ does not have a non-trivial finite $\Lambda_{K / k}$-submodule if and only if

$$
\left|X_{S}(k)\right| \cdot\left|F_{K / k, S}(0)\right|_{p}=p^{\mu_{K / k, S}} .
$$

Proof. By our assumptions, we can show that $X_{S}(K)_{\Gamma} \cong X_{S}(k)$ (see also, e.g., [13, Proposition 2.2.2] for a more general result), and hence $X_{S}(K)_{\Gamma}$ is finite. This implies that $X_{S}(K)^{\Gamma}$ is also finite, and $F_{K / k, S}(0) \neq 0$ (see, e.g., [18, p. 300, Exercise 3]). We can show that $X_{S}(K)$ does not have

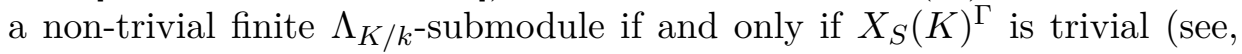
e.g., [18, (5.3.19) Proposition] or the argument given in the proof of $[22$, Proposition 2]). It is known that

$$
\left|X_{S}(K)_{\Gamma}\right| \cdot\left|F_{K / k, S}(0)\right|_{p}=\left|X_{S}(K)^{\Gamma}\right| \cdot p^{\mu_{K / k, S}}
$$

(see, e.g., [18, p. 300, Exercise 3]). The assertion follows from this.

As a corollary, we can obtain the following simpler criterion. (This type result also seems well known. See, e.g., the proof of [23, Theorem 2].)

Corollary 2.2. Let the assumptions be as in Proposition 2.1. If $\left|X_{S}(k)\right|=$ $p$ and $X_{S}(K)$ is infinite, then $X_{S}(K)$ does not have a non-trivial finite $\Lambda_{K / k}$-submodule.

We will prove Theorems 1.1 and 1.2 by using Proposition 2.1 directly. (Note that a similar idea is already used in [4] to show the existence of a non-trivial finite submodule of $X_{S}\left(\mathbb{Q}^{c}\right)$ when $p$ is odd.)

\section{Proof of Theorem 1.1}

In this section, we will only treat the case of $\mathbb{Q}^{c} / \mathbb{Q}$ when $p=2$. Let $S$ be a non-empty finite set of odd prime numbers. In this section, we write $F(T)=F_{\mathbb{Q}^{c} / \mathbb{Q}, S}(T)$ for simplicity (note that $\mu_{\mathbb{Q}^{c} / \mathbb{Q}, S}=0$ ). We can compute $F(T)$ from the results given in [10]. To state this, we need some preparations. 
We define a topological generator of $\operatorname{Gal}\left(\mathbb{Q}^{c} / \mathbb{Q}\right)$ similar to $[10]$. That is, let $\gamma$ be the topological generator of $\operatorname{Gal}\left(\mathbb{Q}\left(\zeta_{4}\right)^{c} / \mathbb{Q}\left(\zeta_{4}\right)\right)$ satisfying $\zeta_{2^{n}}^{\gamma}=\zeta_{2^{n}}^{5}$, and let $\gamma_{1}$ be the restriction of $\gamma$ to $\mathbb{Q}^{c}$. Then $\gamma_{1}$ is a topological generator of $\operatorname{Gal}\left(\mathbb{Q}^{c} / \mathbb{Q}\right)$. We fix an isomorphism $\Lambda_{\mathbb{Q}^{c} / \mathbb{Q}} \rightarrow \mathbb{Z}_{2} \llbracket T \rrbracket$ satisfying $\gamma_{1} \mapsto 1+T$.

We define the subsets $S^{\circ}$ and $S^{\bullet}$ of $S$ by

$$
S^{\circ}=\{q \in S \mid q \equiv 1 \quad(\bmod 4)\}, \quad S^{\bullet}=\{q \in S \mid q \equiv 3 \quad(\bmod 4)\} .
$$

For $q \in S$, we put $P(q)$ the number of primes of $\mathbb{Q}^{c}$ lying above $q$. Let $P^{\circ}$ be the largest number of $P(q)$ for $q \in S^{\circ}$ (if $S^{\circ}$ is empty, we put $P^{\circ}=0$ ). Moreover, let $\mathcal{P}^{\bullet}$ be the set of (distinct) numbers $P(q)$ for $q \in S^{\bullet}$, and put $\mathcal{P}^{\bullet \bullet}=\left\{P \in \mathcal{P}^{\bullet} \mid P \geq P^{\circ}\right\}$ (if $S^{\bullet}$ is empty, then both $\mathcal{P}^{\bullet}$ and $\mathcal{P}^{\bullet \bullet}$ are also empty). We define the following polynomials

$$
F^{\circ}(T)= \begin{cases}\left(\prod_{q \in S^{\circ}}\left((1+T)^{P(q)}-5^{P(q)}\right)\right) /\left((1+T)^{P^{\circ}}-5^{P^{\circ}}\right) & \text { if } S^{\circ} \neq \emptyset \\ 1 & \text { if } S^{\circ}=\emptyset\end{cases}
$$

$$
\begin{aligned}
& F^{\bullet}(T) \\
& = \begin{cases}\left(\prod_{q \in S^{\bullet}}\left((1+T)^{P(q)}+5^{P(q)}\right)\right) /\left(\prod_{P \in \mathcal{P} \bullet \bullet}\left((1+T)^{P}+5^{P}\right)\right) & \text { if } S^{\bullet} \neq \emptyset,\end{cases}
\end{aligned}
$$

Then, from the arguments and results given in [10] (especially, see the proof of Lemma 2.3 of [10]), we see that

$$
F(T)=F^{\circ}(T) \cdot F^{\bullet}(T) .
$$

By using this formula, the value $|F(0)|_{2}$ can be obtained. (Note that $\left|1-5^{2^{a}}\right|_{2}=2^{-(a+2)}$ and $\left|1+5^{2^{a}}\right|_{2}=2^{-1}$.) We can also compute $\left|X_{S}(\mathbb{Q})\right|$ from the following exact sequence

$$
0 \rightarrow E(\mathbb{Q}) \otimes_{\mathbb{Z}} \mathbb{Z}_{2} \rightarrow \bigoplus_{q \in S} R(\mathbb{Q}, q \mathbb{Z}) \rightarrow X_{S}(\mathbb{Q}) \rightarrow 0
$$

and the fact that $E(\mathbb{Q})=\{ \pm 1\}$.

At first, we assume that $S^{\circ}=\emptyset$. In this case, we see that

$$
F(T)=\left(\prod_{q \in S^{\bullet}}\left((1+T)^{P(q)}+5^{P(q)}\right)\right) /\left(\prod_{P \in \mathcal{P} \bullet}\left((1+T)^{P}+5^{P}\right)\right)
$$

From this,

$$
|F(0)|_{2}=2^{|\mathcal{P} \bullet|-|S|} .
$$

We also see that $\left|X_{S}(\mathbb{Q})\right|=2^{|S|-1}$, and hence

$$
\left|X_{S}(\mathbb{Q})\right| \cdot|F(0)|_{2}=2^{|\mathcal{P} \bullet|-1} .
$$


This implies that $\left|X_{S}(\mathbb{Q})\right| \cdot|F(0)|_{2}=1$ if and only if $\left|\mathcal{P}^{\bullet}\right|=1$. When $S=\left\{q_{1}, \ldots, q_{r}\right\},\left|\mathcal{P}^{\bullet}\right|=1$ if and only if $P\left(q_{1}\right)=\cdots=P\left(q_{r}\right)$. Hence, by using Proposition 2.1, the assertion of Theorem 1.1 has been shown for this case.

We shall show the remaining case. It is sufficient to show that $X_{S}\left(\mathbb{Q}^{c}\right)$ has a non-trivial finite submodule when $S^{\circ} \neq \emptyset$. In this case, we see that

$$
\left|F^{\circ}(0)\right|_{2}=2^{2} \cdot P^{\circ} \prod_{q \in S^{\circ}}\left(2^{-2} P(q)^{-1}\right), \quad\left|F^{\bullet}(0)\right|_{2}=2^{\left|\mathcal{P}^{\bullet \bullet}\right|-\left|S^{\bullet}\right|} .
$$

On the other hand, we can show that

$$
\left|X_{S}(\mathbb{Q})\right|=\left(2^{\left|S^{\bullet}\right|} \cdot \prod_{q \in S^{\circ}}\left(2^{2} P(q)\right)\right) / 2 .
$$

Hence

$$
\left|X_{S}(\mathbb{Q})\right| \cdot|F(0)|_{2}=2^{|\mathcal{P} \bullet|+1} \cdot P^{\circ}>1 .
$$

By using Proposition 2.1, we see that $X_{S}\left(\mathbb{Q}^{c}\right)$ has a non-trivial finite submodule in this case. Thus we have completed the proof of Theorem 1.1.

\section{Totally real fields}

We shall show Theorem 1.2, however, we will give a simple remark before this.

Remark 4.1. Let $k$ be a real quadratic field, and $p$ an odd prime number. Let $S$ be a non-empty finite set of prime numbers not containing $p$. For the structure of $X_{S}\left(k^{c}\right)$, it is sufficient to consider the case that every $q \in S$ satisfies either

(a) $q \equiv 1(\bmod p)$, or

(b) $q \equiv-1(\bmod p)$ and $q$ is inert in $k$

(see $[8])$. We put

$$
S_{1}=\{q \in S \mid q \equiv 1 \quad(\bmod p)\} .
$$

We note that if $S_{1} \neq \emptyset$, then $X_{S}\left(k^{c}\right)$ always contains a non-trivial finite

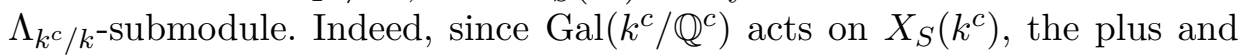
minus parts

$$
X_{S}\left(k^{c}\right)^{ \pm}=\left\{x \in X_{S}\left(k^{c}\right) \mid \sigma(x)= \pm x \text { for the generator } \sigma \text { of } \operatorname{Gal}\left(k^{c} / \mathbb{Q}^{c}\right)\right\}
$$

can be defined, and we see that $X_{S}\left(k^{c}\right) \cong X_{S}\left(k^{c}\right)^{+} \oplus X_{S}\left(k^{c}\right)^{-}$. We can show that $X_{S}\left(k^{c}\right)^{+}$is isomorphic to $X_{S_{1}}\left(\mathbb{Q}^{c}\right)$, and this is not trivial because $S_{1} \neq \emptyset$ (see also [10]). Hence, the assertion follows from the fact (which is shown in [4]) that $X_{S_{1}}\left(\mathbb{Q}^{c}\right)$ contains a non-trivial finite submodule. (The same type result for imaginary quadratic fields is given in [4].) 
Proof of Theorem 1.2. Let $k$ be a real quadratic field. Assume that $p$ is inert in $k$, and $p(>2)$ does not divide the class number of $k$. Let $k_{n}^{c}$ be the $n$th layer of $k^{c} / k$. Take a topological generator $\gamma$ of $\operatorname{Gal}\left(k\left(\zeta_{p}\right)^{c} / k\left(\zeta_{p}\right)\right)$ which satisfies $\zeta_{p^{n}}^{\gamma}=\zeta_{p^{n}}^{1+p}$ for all $n$. Let $\gamma_{1}$ be the restriction of $\gamma$ to $k^{c}$, then $\gamma_{1}$ is a topological generator of $\operatorname{Gal}\left(k^{c} / k\right)$. We fix an isomorphism from $\Lambda_{k^{c} / k}$ to $\mathbb{Z}_{p} \llbracket T \rrbracket$ satisfying $\gamma_{1} \mapsto 1+T$.

Let $q_{1}, \ldots, q_{r}$ be distinct prime numbers satisfying the assumption of this theorem. For each $i$, we see that $R\left(k, q_{i} O_{k}\right)$ is a cyclic group of order $p \cdot P\left(q_{i}\right)$. Since $X_{\emptyset}(k)=0$, we obtain the following exact sequence

$$
E(k) \otimes_{\mathbb{Z}} \mathbb{Z}_{p} \rightarrow \bigoplus_{i=1}^{r} R\left(k, q_{i} O_{k}\right) \rightarrow X_{S}(k) \rightarrow 0 .
$$

We note that $E(k) \otimes_{\mathbb{Z}} \mathbb{Z}_{p}$ is a cyclic $\mathbb{Z}_{p}$-module. Hence, we have the inequalities

$$
p^{r-1} \cdot\left(\prod_{i=1}^{r} P\left(q_{i}\right)\right) / P^{\prime} \leq\left|X_{S}(k)\right| \leq p^{r} \cdot \prod_{i=1}^{r} P\left(q_{i}\right) .
$$

We will compute the characteristic polynomial of $X_{S}\left(k^{c}\right)$. The following argument is essentially given in [8, Section 6], however, we shall reconstruct it for our situation. We denote by $k_{n}^{c}$ the $n$th layer of $k^{c} / k$. We put $R_{i}=\lim _{n} R\left(k_{n}^{c}, q_{i} O_{k_{n}^{c}}\right.$ ) (for each $i$ ) and $\mathcal{E}=\lim _{n} E\left(k_{n}^{c}\right) \otimes_{\mathbb{Z}} \mathbb{Z}_{p}$, where the projective limits are taken with respect to the norm mappings. By using class field theory, we obtain the following exact sequence

$$
\mathcal{E} \rightarrow \bigoplus_{i=1}^{r} R_{i} \rightarrow X_{S}\left(k^{c}\right) \rightarrow 0
$$

of $\mathbb{Z}_{p} \llbracket \operatorname{Gal}\left(k^{c} / \mathbb{Q}\right) \rrbracket$-modules (note that $X_{\emptyset}\left(k^{c}\right)=0$ by Iwasawa's result [11]). For each term of the above exact sequence, we can consider its plus and minus parts with respect to the action of $\operatorname{Gal}\left(k^{c} / \mathbb{Q}^{c}\right)$. We note that $X_{S}\left(k^{c}\right)^{+} \cong$ $X_{S}\left(\mathbb{Q}^{c}\right)=0$ because $q_{i} \equiv-1(\bmod p)$ for all $i$ (see also Remark 4.1$)$. Hence we will consider the structure of $X_{S}\left(k^{c}\right)^{-}\left(\cong X_{S}\left(k^{c}\right)\right)$ as a module over $\Lambda_{k^{c} / k} \cong \mathbb{Z}_{p} \llbracket T \rrbracket$. We can show that

$$
R_{i}^{-} \cong R_{i} \cong \mathbb{Z}_{p} \llbracket T \rrbracket /\left((1+T)^{P\left(q_{i}\right)}-(1+p)^{P\left(q_{i}\right)}\right)
$$

as $\mathbb{Z}_{p} \llbracket T \rrbracket$-modules for each $i$ (see, e.g., the argument given in the proof of $\left[10\right.$, Lemma 2.1]). Note also that $\mathcal{E}^{-} \cong \mathbb{Z}_{p} \llbracket T \rrbracket$ as a $\mathbb{Z}_{p} \llbracket T \rrbracket$-module. (For example, by using $[18,(11.3 .11)$ Theorem (iii)], we see that

$$
\mathcal{E} \cong \mathbb{Z}_{p} \llbracket \operatorname{Gal}\left(k^{c} / \mathbb{Q}\right) \rrbracket \cong \mathbb{Z}_{p}[\operatorname{Gal}(k / \mathbb{Q})] \llbracket T \rrbracket,
$$

and hence the fact follows.) From [8, Theorem 1.1], we see that $X_{\left\{q_{i}\right\}}\left(k^{c}\right)$ is finite for each $i$. Hence, by using the same type argument given in [10], 
we obtain the following exact sequence

$$
0 \rightarrow \mathcal{E}^{-} /\left((1+T)^{P^{\prime}}-(1+p)^{P^{\prime}}\right) \rightarrow \bigoplus_{i=1}^{r} R_{i} \rightarrow X_{S}\left(k^{c}\right) \rightarrow 0
$$

as $\mathbb{Z}_{p} \llbracket T \rrbracket$-modules. From the above results, we can see that

$$
F_{k^{c} / k, S}(T)=\left(\prod_{i=1}^{r}\left((1+T)^{P\left(q_{i}\right)}-(1+p)^{P\left(q_{i}\right)}\right)\right) /\left((1+T)^{P^{\prime}}-(1+p)^{P^{\prime}}\right)
$$

(and $\mu_{k^{c} / k, S}=0$ ). Then, we can obtain the formula

$$
\left|F_{k^{c} / k, S}(0)\right|_{p}=p^{1-r} \cdot P^{\prime} /\left(\prod_{i=1}^{r} P\left(q_{i}\right)\right) .
$$

Consequently, we see that $\left|X_{S}(k)\right| \cdot\left|F_{k^{c} / k, S}(0)\right|_{p}=1$ if and only if

$$
\left|X_{S}(k)\right|=p^{r-1} \cdot\left(\prod_{i=1}^{r} P\left(q_{i}\right)\right) / P^{\prime}
$$

The assertion follows from Proposition 2.1.

As a special case of Theorem 1.2, we obtain the following:

Corollary 4.2. Let the assumptions be as in Theorem 1.2, and suppose also that $P\left(q_{i}\right)=1$ for $i=1, \ldots, r$. Then, $X_{S}\left(k^{c}\right)$ does not have a non-trivial finite submodule if and only if $\left|X_{S}(k)\right|=p^{r-1}$.

Remark 4.3. Let the assumptions be as in the above corollary (that is, $P\left(q_{i}\right)=1$ for all $\left.i\right)$. In this case, we can show that if $\left|X_{\left\{q_{i}\right\}}(k)\right|=1$ for some $q_{i}$, then $\left|X_{S}(k)\right|=p^{r-1}$, and hence $X_{S}\left(k^{c}\right)$ does not have a non-trivial finite submodule. We will give an example. In the case when $k=\mathbb{Q}(\sqrt{2})$ and $p=3$, it can be shown that $\left|X_{\{q\}}(k)\right|=1$ for $q=5,11,83$. (The author used PARI/GP [25] (versions 2.9.1 and 2.9.3) to check these examples.) As a consequence, at least for $k=\mathbb{Q}(\sqrt{2})$ and $p=3$, we can take a set $S$ such that $X_{S}\left(k^{c}\right) \cong \mathbb{Z}_{3}^{\oplus c}$ (as a $\mathbb{Z}_{3}$-module) for any given positive integer $c$ (e.g., $\left.X_{\{5,11,29\}}\left(k^{c}\right) \cong \mathbb{Z}_{3}^{\oplus 2}\right)$.

Remark 4.4. Let the assumptions be as in Theorem 1.2, however, we remove the assumption that $p$ does not divide the class number of $k$. We also assume that $X_{\emptyset}\left(k^{c}\right)$ is non-trivial and finite. Under these assumptions, we can see that the characteristic polynomial $F_{k^{c} / k, S}(T)$ of $X_{S}\left(k^{c}\right)$ is the same as in the proof of Theorem 1.2. In this case, we see that

$$
\left|X_{S}(k)\right| \cdot\left|F_{k^{c} / k, S}(0)\right|_{p} \geq\left|X_{\emptyset}(k)\right|>1
$$

(recall also that $\mu_{k^{c} / k, S}=0$ ), and hence $X_{S}\left(k^{c}\right)$ contains a non-trivial finite submodule. 
We will give another example. The method of construction is different from Theorem 1.2.

Proposition 4.5. Let $p$ be an odd prime number. There is a finite set $S$ of prime numbers (not containing $p$ ), and a finite p-extension $k$ of $\mathbb{Q}$ such that $X_{S}\left(k^{c}\right) \cong \mathbb{Z}_{p}$ as a $\mathbb{Z}_{p}$-module.

Proof. We use the result given in [17] (see also [9]). Let $S=\left\{q_{1}, q_{2}\right\}$ be a set of distinct prime numbers satisfying the condition of [17, Theorem 1]. (We will not use this condition directly in this proof. For the existence of such a set $S$, see also $\left[17\right.$, Remark 1 , Remark 2].) Let $\mathcal{L}_{S}\left(\mathbb{Q}^{c}\right) / \mathbb{Q}^{c}$ be the maximal pro- $p$ extension unramified outside $S$. From [17, Theorem 1], we see that $\operatorname{Gal}\left(\mathcal{L}_{S}\left(\mathbb{Q}^{c}\right) / \mathbb{Q}^{c}\right)$ is isomorphic to an infinite metacyclic pro- $p$ group $G$ topologically generated by $a, b$ which satisfy

$$
a^{p^{2}}=1, \quad b^{-1} a b=a^{1+p} .
$$

(In the following, we will identify $\operatorname{Gal}\left(\mathcal{L}_{S}\left(\mathbb{Q}^{c}\right) / \mathbb{Q}^{c}\right)$ with $G$.) For a positive integer $n$, let $H_{n}$ be the open subgroup of $G$ which is topologically generated by $b^{p^{n}}$. Then, we can take $n$ such that the fixed field $L$ of $\mathcal{L}_{S}\left(\mathbb{Q}^{c}\right)$ by $H_{n}$ is a Galois extension over $\mathbb{Q}$. Since $L / \mathbb{Q}^{c}$ is finite, there is a finite $p$-extension $k / \mathbb{Q}$ such that $L=k^{c}$. We also note that $k$ is totally real.

By the above results, $\operatorname{Gal}\left(\mathcal{L}_{S}\left(\mathbb{Q}^{c}\right) / k^{c}\right) \cong \mathbb{Z}_{p}$. Note that $\mathcal{L}_{S}\left(\mathbb{Q}^{c}\right)$ is also the maximal pro- $p$ extension of $k^{c}$ unramified outside $S$. This implies that $X_{S}\left(k^{c}\right) \cong \operatorname{Gal}\left(\mathcal{L}_{S}\left(\mathbb{Q}^{c}\right) / k^{c}\right)$. The assertion follows.

\section{Proof of Theorem 1.3}

In this section, we put $k=\mathbb{Q}(\sqrt{-3})$ and $p=3$. Note that there is only one prime $\mathfrak{p}$ of $k$ lying above 3 . Suppose that $q_{1}, q_{2}$ satisfy the assumptions of Theorem 1.3, and put $S=\left\{q_{1}, q_{2}\right\}$. We see that $q_{i}$ is inert in $k$ and $\left|R\left(k, q_{i} O_{k}\right)\right|=3$ for $i=1,2$. Note also that $\left|X_{S}(k)\right|=3$ because the image of $\zeta_{3}$ in $R\left(k, q_{1} O_{k}\right) \oplus R\left(k, q_{2} O_{k}\right)$ is not trivial.

First, we will show that $X_{S}(\widetilde{k})$ is not pseudo-null. To see this, we need some preparations. Let $k^{a} / k$ be the anti-cyclotomic $\mathbb{Z}_{3}$-extension, and $k_{m}^{a}$ its $m$ th layer.

Lemma 5.1. Let the assumptions be as in Theorem 1.3. We put $F=k_{m}^{a}$. Then $\operatorname{dim}_{\mathbb{Q}_{3}} X_{S}\left(F^{c}\right) \otimes_{\mathbb{Z}_{3}} \mathbb{Q}_{3} \geq 3^{m}-1$.

Proof. Our proof of this lemma uses a method given in [8], [10], [17], etc.

Take a topological generator $\gamma$ of $\operatorname{Gal}\left(F^{c} / F\right)$ satisfying $\zeta_{3^{n}}^{\gamma}=\zeta_{3^{n}}^{4}$ for all $n$, and fix an isomorphism from $\Lambda_{F^{c} / F}$ to $\mathbb{Z}_{p} \llbracket T \rrbracket$ satisfying $\gamma \mapsto 1+T$.

We remark that the prime of $k$ lying above $q_{i}$ splits completely in $F$ (see, e.g., [12]), and hence there are $3^{m}$ primes in $F$ lying above $q_{i}$ (for $i=1,2$ ). Put $r=3^{m}$. We denote by $\mathfrak{q}_{1,1}, \ldots, \mathfrak{q}_{r, 1}$ (resp. $\left.\mathfrak{q}_{1,2}, \ldots, \mathfrak{q}_{r, 2}\right)$ the primes of $F$ lying above $q_{1}$ (resp. $q_{2}$ ). Note that each $\mathfrak{q}_{i, j}$ is not decomposed in $F^{c} / F$ 
by the assumptions. Let $F_{n}^{c}$ be the $n$th layer of $F^{c} / F$. We denote by $i_{n}\left(\mathfrak{q}_{i, j}\right)$ the extension of $\mathfrak{q}_{i, j}$ in $F_{n}^{c}$. Note that we can see that

$$
{\underset{\varliminf}{n}}_{\lim _{n}} R\left(F_{n}^{c}, i_{n}\left(\mathfrak{q}_{i, j}\right)\right) \cong \mathbb{Z}_{3} \llbracket T \rrbracket /(T-3)
$$

as a $\mathbb{Z}_{3} \llbracket T \rrbracket$-module (see also the proof of Theorem 1.2 in Section 4 ).

Note that 3 does not divide the class number of $k$, and only $\mathfrak{p}$ is ramified in $F_{n}^{c} / k$. Hence $X_{\emptyset}\left(F^{c}\right)$ is trivial (by Iwasawa's result [11]). We put $\mathcal{E}=$ $\lim _{n} E\left(F_{n}^{c}\right) \otimes_{\mathbb{Z}} \mathbb{Z}_{3}$ where the projective limit is taken with respect to the norm mappings. We can also regard $\mathcal{E}$ as a $\mathbb{Z}_{3} \llbracket T \rrbracket$-module. By using class field theory, we obtain the following exact sequence

$$
\mathcal{E} /(T-3) \rightarrow \lim _{n} \bigoplus_{j=1}^{2} \bigoplus_{i=1}^{r} R\left(F_{n}^{c}, i_{n}\left(\mathfrak{q}_{i, j}\right)\right) \rightarrow X_{S}\left(F^{c}\right) \rightarrow 0
$$

(cf. e.g., [17], [10]). Note that the second term is isomorphic to $\left(\mathbb{Z}_{p} \llbracket T \rrbracket /\right.$ $(T-3))^{\oplus 2 r}$, and hence it is free of rank $2 r$ as a $\mathbb{Z}_{3}$-module.

By using [18, (11.3.11) Theorem (iii)] ( $F^{c} / F$ satisfies the assumption of this theorem), we see that

$$
\mathcal{E} \cong \mathbb{Z}_{3} \llbracket T \rrbracket^{\oplus r} \oplus \mathbb{Z}_{3}(1)
$$

here $\mathbb{Z}_{3}(1)$ is the first Tate twist of $\mathbb{Z}_{3}$. Hence $\operatorname{dim}_{\mathbb{Q}_{3}} \mathcal{E} /(T-3) \otimes_{\mathbb{Z}_{3}} \mathbb{Q}_{3}=r+1$.

The assertion follows from these facts.

Lemma 5.2. Under the assumptions of Theorem 1.3, $X_{S}(\widetilde{k})$ is not pseudonull as a $\Lambda_{\widetilde{k} / k}$-module.

Proof. Let the notations be as in Lemma 5.1. Note that $F^{c}$ is an intermediate field of $\widetilde{k} / k^{c}$, and $F^{c} / k^{c}$ is a cyclic extension of degree $3^{m}$. Since $\mathfrak{p}$ is totally ramified in $\widetilde{k} / k$, we see that the $\operatorname{Gal}\left(\widetilde{k} / F^{c}\right)$-coinvariant quotient $X_{S}(\widetilde{k})_{\operatorname{Gal}\left(\widetilde{k} / F^{c}\right)}$ is isomorphic to $X_{S}\left(F^{c}\right)$. We can also show that $X_{S}(\widetilde{k})$ is a finitely generated $\mathbb{Z}_{3} \llbracket \operatorname{Gal}\left(\widetilde{k} / k^{c}\right) \rrbracket$-module because $X_{S}(\widetilde{k})_{\operatorname{Gal}\left(\widetilde{k} / k^{c}\right)} \cong X_{S}\left(k^{c}\right)$ is finitely generated over $\mathbb{Z}_{3}$ (see, e.g., [10]). However, Lemma 5.1 implies that $X_{S}(\widetilde{k})$ is not a torsion $\mathbb{Z}_{3} \llbracket \operatorname{Gal}\left(\widetilde{k} / k^{c}\right) \rrbracket$-module. From this, we can deduce that $X_{S}(\widetilde{k})$ is not pseudo-null as a $\Lambda_{\widetilde{k} / k}$-module (see Greenberg [7], or Lemma 2.3 of Fujii [3]).

Remark 5.3. Concerning the non-pseudo-nullity of $X_{S}(\tilde{k})$ (for a general imaginary quadratic field $k$ ), see also Kataoka [14]. However, Kataoka's result does not cover our case $(p=3$ and $k=\mathbb{Q}(\sqrt{-3}))$.

Lemma 5.4. Under the assumptions of Theorem 1.3, $X_{S}(K)$ does not have a non-trivial finite $\Lambda_{K / k}$-submodule for every $\mathbb{Z}_{3}$-extension $K / k$. 
Proof. Let $K / k$ be an arbitrary $\mathbb{Z}_{3}$-extension, and put $H=\operatorname{Gal}(\widetilde{k} / K)$. We recall that $\mathfrak{p}$ is totally ramified in $K / k$, and $\left|X_{S}(k)\right|=3$. Hence, by Corollary 2.2, it is sufficient to show that $X_{S}(K)$ is infinite. Assume that $X_{S}(K)$ is finite. In our situation, the $H$-coinvariant quotient $X_{S}(\widetilde{k})_{H}$ is isomorphic to $X_{S}(K)$, and hence $X_{S}(\widetilde{k})_{H}$ is also finite. Then we can see that $X_{S}(\widetilde{k})$ is pseudo-null by using Perrin-Riou's result [24, Lemme 4] (see also Minardi [15]). However, this contradicts to Lemma 5.2.

The remaining part of our proof of Theorem 1.3 is heavily relied on Greenberg's results given in [6]. Assume that the maximal pseudo-null submodule $Z$ of $X_{S}(\widetilde{k})$ is not trivial. Let $I$ be the augmentation ideal of $\mathbb{Z}_{3} \llbracket \operatorname{Gal}(\widetilde{k} / k) \rrbracket$. We claim that $Z / I$ is finite. To show this, we use a similar argument which is given in the paragraph before Lemma 5 of [6]. Let $K / k$ be a $\mathbb{Z}_{3}$-extension. We put $H=\operatorname{Gal}(\widetilde{k} / K)$ and $\Gamma=\operatorname{Gal}(K / k)$. Recall that $\mathfrak{p}$ is totally ramified in $\widetilde{k} / k$. Then $X_{S}(\widetilde{k})_{H} \cong X_{S}(K)$, and it is a finitely generated torsion $\mathbb{Z}_{3} \llbracket \operatorname{Gal}(K / k) \rrbracket$-module. From this, we can see that $\left(X_{S}(\widetilde{k}) / Z\right)^{H}$ is trivial, and hence the natural $\mathbb{Z}_{3} \llbracket \mathrm{Gal}(K / k) \rrbracket$-module homomorphism $Z_{H} \rightarrow X_{S}(\widetilde{k})_{H}\left(\cong X_{S}(K)\right)$ is injective. Moreover, since $X_{S}(K)_{\Gamma} \cong X_{S}(k)$ is finite, we can show that $\left(Z_{H}\right)_{\Gamma}$ is finite. Then the claim follows. From this, we can apply [6, Lemma 5]. In this case, there must be a $\mathbb{Z}_{3}$-extension $K^{\dagger} / k$ such that $Z_{\mathrm{Gal}\left(K^{\dagger} / k\right)}$ has a non-trivial finite submodule, and then $X_{S}\left(K^{\dagger}\right)$ has a non-trivial finite submodule from the above argument. This contradicts Lemma 5.4. Hence, Theorem 1.3 completely follows.

Remark 5.5. There is another method to deduce Theorem 1.3 from the lemmas. We shall state this briefly. We take the isomorphism $\mathbb{Z}_{3} \llbracket \operatorname{Gal}\left(k^{c} / k\right) \rrbracket \cong \mathbb{Z}_{3} \llbracket T \rrbracket$ given in the proof of Lemma 5.1 , then we see that $\mu_{k^{c} / k, S}=0$ and $F_{k^{c} / k, S}(T)=T-3$ (see, e.g., [10]). Moreover, by Lemma 5.4 , we see that $X_{S}\left(k^{c}\right) \cong \mathbb{Z}_{3}$ as a $\mathbb{Z}_{3}$-module. Recall also that $X_{S}(\widetilde{k})_{\operatorname{Gal}\left(\widetilde{k} / k^{c}\right)} \cong X_{S}\left(k^{c}\right)$. Moreover, it can be shown that $X_{S}(\widetilde{k})$ is a cyclic $\mathbb{Z}_{3} \llbracket \operatorname{Gal}(\widetilde{k} / k) \rrbracket$-module. Hence, $X_{S}(\widetilde{k}) \cong \mathbb{Z}_{3} \llbracket \operatorname{Gal}(\widetilde{k} / k) \rrbracket / \mathfrak{A}$, where $\mathfrak{A}$ is the annihilator ideal of $\mathbb{Z}_{3} \llbracket \operatorname{Gal}(\tilde{k} / k) \rrbracket$ for $X_{S}(\widetilde{k})$. By Lemma $5.2, X_{S}(\widetilde{k})$ is not pseudo-null. By a similar argument given in the proof of [2, Proposition 3.1] (or $[15$, Section 3.D]), we can show that $\mathfrak{A}$ is a principal ideal generated by an irreducible element. (The fact that $F_{k^{c} / k, S}(T)=T-3$ is crucial.) Hence, $X_{S}(\widetilde{k})$ does not contain a non-trivial pseudo-null submodule.

\section{Appendix A. Weak analogs of Greenberg's conjecture}

Let $k$ be a totally real field and $p$ an odd prime number. We denote by $S$ a finite set of prime numbers which does not contain $p$. To consider 
the structure of $X_{S}\left(k^{c}\right)$, it is sufficient to treat $S$ satisfying the following condition (see also, e.g., [10]):

(R) For each $q \in S$, there is a prime $\mathfrak{q}$ of $k$ lying above $q$ such that $R(k, \mathfrak{q})$ is not trivial.

When $k$ is a real quadratic field, the condition $(\mathrm{R})$ is equivalent to the condition that every $q \in S$ satisfies (a) or (b) in Remark 4.1.

Let $M_{S, p}\left(k^{c}\right)$ be the maximal abelian pro- $p$ extension of $k^{c}$ unramified outside $S \cup\{p\}$. In this case, we see that $\operatorname{Gal}\left(M_{S, p}\left(k^{c}\right) / k^{c}\right)$ is a finitely

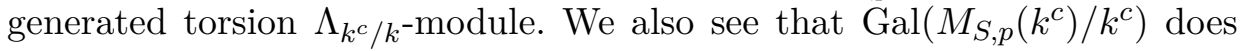

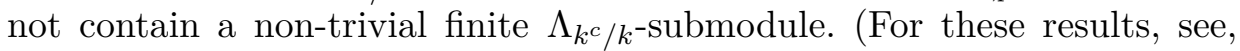
e.g., [6], [18].)

First, we consider the case when $S=\emptyset$. It is conjectured that $X_{\emptyset}\left(k^{c}\right)$ is finite (Greenberg's conjecture [5]). Moreover, some weak forms of this conjecture are also proposed (see Nguyen Quang Do [19], [20]).

(Conj1) $X_{\emptyset}\left(k^{c}\right)$ is trivial or contains a non-trivial finite $\Lambda_{k^{c}} / k^{\text {-submodule. }}$ $(\operatorname{Conj2}) \operatorname{Gal}\left(M_{\emptyset, p}\left(k^{c}\right) / k^{c}\right)$ is trivial or $\operatorname{Gal}\left(M_{\emptyset, p}\left(k^{c}\right) / L_{\emptyset}\left(k^{c}\right)\right)$ is not trivial.

Remark A.1. Note that (Conj1) implies (Conj2). If $p$ splits completely in $k$ and Leopoldt's conjecture holds for $k$ and $p$, then (Conj1) and (Conj2) are equivalent (see [19], [22]). In [19] and [20], Nguyen Quang Do considered these conjectures for the case when $k$ is a real abelian field and $p$ splits completely in $k$ (see also [21]).

Next, we shall consider the " $S$-ramified" version of these assertions:

(Conj1S) $X_{S}\left(k^{c}\right)$ is trivial or contains a non-trivial finite $\Lambda_{k^{c}} / k^{\text {-submodule. }}$ $\left(\right.$ Conj2S) $\operatorname{Gal}\left(M_{S, p}\left(k^{c}\right) / k^{c}\right)$ is trivial or $\operatorname{Gal}\left(M_{S, p}\left(k^{c}\right) / L_{S}\left(k^{c}\right)\right)$ is not trivial.

However, the results given in Section 4 imply that the assertion (Conj1S) does not hold in general. For the assertion (Conj2S), we can obtain the following:

Theorem A.2. Let $k$ be a totally real field, $p$ an odd prime number, and $S$ a non-empty finite set of prime numbers which does not contain $p$. Assume that $S$ satisfies $(\mathrm{R})$. If $\operatorname{Gal}\left(L_{\{q\}}\left(k^{c}\right) / L_{\emptyset}\left(k^{c}\right)\right)$ is finite for some $q \in S$, then $\operatorname{Gal}\left(M_{S, p}\left(k^{c}\right) / L_{S}\left(k^{c}\right)\right)$ is not trivial.

Proof. When $k=\mathbb{Q}$, this assertion is already mentioned in [4] (and the proof of our case is almost similar). Take a prime number $q \in S$ such that $\operatorname{Gal}\left(L_{\{q\}}\left(k^{c}\right) / L_{\emptyset}\left(k^{c}\right)\right)$ is finite. Since $S$ satisfies $(\mathrm{R})$, we see that $\operatorname{Gal}\left(M_{\{q\}, p}\left(k^{c}\right) / M_{\emptyset, p}\left(k^{c}\right)\right)$ is infinite (see, e.g., [18, (11.3.5) Theorem and (11.3.6) Corollary]). Hence $\operatorname{Gal}\left(M_{\{q\}, p}\left(k^{c}\right) / L_{\emptyset}\left(k^{c}\right)\right)$ is also infinite. Thus, we conclude that $\operatorname{Gal}\left(M_{\{q\}, p}\left(k^{c}\right) / L_{\{q\}}\left(k^{c}\right)\right)$ is infinite. Since $L_{S}\left(k^{c}\right) \subseteq$ $M_{\{q\}, p}\left(k^{c}\right) L_{S}\left(k^{c}\right) \subseteq M_{S, p}\left(k^{c}\right)$, we can show that $\operatorname{Gal}\left(M_{S, p}\left(k^{c}\right) / L_{S}\left(k^{c}\right)\right)$ is not trivial. (Note that we do not need the validity of Leopoldt's conjecture in this proof.) 
Moreover, we can also see the following:

Proposition A.3. Let $k$ be a totally real field, $p$ an odd prime number, and $S$ a non-empty finite set of prime numbers which does not contain $p$. Assume that Leopoldt's conjecture holds for $k$ and $p$. Assume also that $p$ splits completely in $k$. Then $X_{S}\left(k^{c}\right)$ contains a non-trivial finite $\Lambda_{k^{c}} / k^{-}$ submodule if and only if $\operatorname{Gal}\left(M_{S, p}\left(k^{c}\right) / L_{S}\left(k^{c}\right)\right)$ is not trivial.

Proof. The proof is quite similar to the case when $S=\emptyset$. See [22] or [19] (see also [4] for the case when $k=\mathbb{Q}$ and $S \neq \emptyset$ ). Note that we need the finiteness of $\operatorname{Gal}\left(M_{S, p}\left(k^{c}\right) / k^{c}\right)_{\Gamma}$ (where $\left.\Gamma=\operatorname{Gal}\left(k^{c} / k\right)\right)$ to show the triviality of $\operatorname{Gal}\left(M_{S, p}\left(k^{c}\right) / k^{c}\right)^{\Gamma}$, however, this follows from class field theory and the validity of Leopoldt's conjecture for $k$ and $p$ (see, e.g., [18]).

Hence we obtained the following:

Corollary A.4. Let $k$ be a totally real field, $p$ an odd prime number, and $S$ a non-empty finite set of prime numbers which does not contain p. Assume that $S$ satisfies (R). Assume also that p splits completely in $k$, and Leopoldt's conjecture holds for $k$ and $p$. If $\operatorname{Gal}\left(L_{\{q\}}\left(k^{c}\right) / L_{\emptyset}\left(k^{c}\right)\right)$ is finite for some $q \in S$, then $X_{S}\left(k^{c}\right)$ contains a non-trivial finite submodule.

When $k$ is a real abelian field and $p$ is an odd prime, it was shown that $\operatorname{Gal}\left(L_{\{q\}}\left(k^{c}\right) / L_{\emptyset}\left(k^{c}\right)\right)$ is finite for every prime number $q(\neq p)$ (see $[8$, Theorem 1.1]). Hence, by combining the validity of Leopoldt's conjecture (see [1]), we can also obtain the following:

Corollary A.5. Let $k$ be a real abelian field, $p$ an odd prime number, and $S$ a non-empty finite set of prime numbers which does not contain $p$. Assume that $S$ satisfies (R). Then the following statements hold.

(1) $\operatorname{Gal}\left(M_{S, p}\left(k^{c}\right) / L_{S}\left(k^{c}\right)\right)$ is not trivial.

(2) Moreover, if $p$ splits completely in $k$, then $X_{S}\left(k^{c}\right)$ contains a nontrivial finite submodule.

Note that the assertions of the above corollary were already shown in [4] for the case when $k=\mathbb{Q}$.

\section{References}

[1] A. Brumer, "On the units of algebraic number fields", Mathematika, Lond. 14 (1967), no. 2, p. 121-124.

[2] S. FujII, "Pseudo-null submodules of the unramified Iwasawa module for $\mathbb{Z}_{p}^{2}$-extensions", Interdiscip. Inf. Sci. 16 (2010), no. 1, p. 55-66.

[3] - "On the depth of the relations of the maximal unramified pro- $p$ Galois group over the cyclotomic $\mathbb{Z}_{p}$-extension", Acta Arith. 149 (2011), no. 2, p. 101-110.

[4] S. Fuji \& T. Ітон, "Some remarks on pseudo-null submodules of tamely ramified Iwasawa modules", J. Théor. Nombres Bordx 30 (2018), no. 2, p. 533-555.

[5] R. Greenberg, "On the Iwasawa invariants of totally real number fields", Am. J. Math. 98 (1976), no. 1, p. 263-284. 
[6] — "On the structure of certain Galois groups", Invent. Math. 47 (1978), no. 1, p. 85-99.

[7] — , "Iwasawa theory - past and present", in Class field theory - its centenary and prospect, Advanced Studies in Pure Mathematics, vol. 30, Mathematical Society of Japan, 2001, p. 335-385.

[8] Т. Ітон, "On tamely ramified Iwasawa modules for the cyclotomic $\mathbb{Z}_{p}$-extension of abelian fields", Osaka J. Math. 51 (2014), no. 2, p. 513-536.

[9] T. Iтон \& Y. Mizusawa, "On tamely ramified pro-p-extensions over $\mathbb{Z}_{p}$-extensions of $\mathbb{Q}$ ", Math. Proc. Camb. Philos. Soc. 156 (2014), no. 2, p. 281-294.

[10] T. Ітон, Y. Mizusawa \& M. Ozaki, "On the $\mathbb{Z}_{p}$-ranks of tamely ramified Iwasawa modules", Int. J. Number Theory 9 (2013), no. 6, p. 1491-1503.

[11] K. Iwasawa, "A note on class numbers of algebraic number fields", Abh. Math. Semin. Univ. Hamb. 20 (1956), no. 3-4, p. 257-258.

[12] - "On the $\mu$-invariants of $\boldsymbol{Z}_{l}$-extensions", in Number theory, algebraic geometry and commutative algebra, in honor of Yasuo Akizuki, Kinokuniya Book-Store Co., 1973, p. 1-11.

[13] J.-F. Jaulent, C. Maire \& G. Perbet, "Sur les formules asymptotiques le long des $\mathbb{Z}_{\ell^{-}}$ extensions", Ann. Math. Qué. 37 (2013), no. 1, p. 63-78.

[14] T. KATAOKA, "On pseudo-isomorphism classes of tamely ramified Iwasawa modules over imaginary quadratic fields", Acta Arith. 180 (2017), no. 2, p. 171-182.

[15] J. Minardi, "Iwasawa modules for $\mathbb{Z}_{p}^{d}$-extensions of algebraic number fields", PhD Thesis, University of Washington (USA), 1986.

[16] Y. Mizusawa, "Tame pro-2 Galois groups and the basic $\mathbb{Z}_{2}$-extension", Trans. Am. Math. Soc. 370 (2018), no. 4, p. 2423-2461.

[17] Y. Mizusawa \& M. Ozaki, "On tame pro- $p$ Galois groups over basic $\mathbb{Z}_{p}$-extensions", Math. Z. 273 (2013), no. 3-4, p. 1161-1173.

[18] J. Neukirch, A. Schmidt \& K. Wingberg, Cohomology of number fields, 2nd ed., Grundlehren der Mathematischen Wissenschaften, vol. 323, Springer, 2008.

[19] T. NGuyen Quang Do, "Sur la conjecture faible de Greenberg dans le cas abélien $p$ décomposé", Int. J. Number Theory 2 (2006), no. 1, p. 49-64.

[20] - "Sur une forme faible de la conjecture de Greenberg II", Int. J. Number Theory 13 (2017), no. 4, p. 1061-1070.

[21] - "Formules de genres et conjecture de Greenberg", Ann. Math. Qué. 42 (2018), no. 2, p. 267-280.

[22] M. OzAKI, "The class group of $\boldsymbol{Z}_{p}$-extensions over totally real number fields", Tôhoku Math. J. 49 (1997), no. 3, p. 431-435.

[23] - "On the cyclotomic unit group and the ideal class group of a real abelian number field II", J. Number Theory 64 (1997), no. 2, p. 223-232.

[24] B. Perrin-Riou, "Arithmétique des courbes elliptiques et théorie d'Iwasawa", Mém. Soc. Math. Fr., Nouv. Sér. 17 (1984), p. 1-130.

[25] The PARI Group, "PARI/GP version 2.9.3", 2017, available from http://pari.math. u-bordeaux.fr/.

[26] L. C. Washington, Introduction to cyclotomic fields, Graduate Texts in Mathematics, vol. 83, Springer, 1997.

Tsuyoshi Ітон

Division of Mathematics, Education Center

Faculty of Social Systems Science

Chiba Institute of Technology

2-1-1 Shibazono, Narashino

Chiba, 275-0023, Japan

E-mail: tsuyoshi.itoh@it-chiba.ac.jp 